\title{
Prediction of the course of primary open-angle glaucoma in combination with diabetic retinopathy using a mathematical model
}

\author{
I $V$ Vorobyeva ${ }^{1,}$ \\ ${ }^{1}$ Russian Medical Academy of Continuous Professional Education, 2/1, Barrikadnaya \\ Street, Moscow, 125993, Russia
}

\begin{abstract}
It is possible to accurately diagnose diseases in the early stages using optical coherence tomography. A mathematical model has been developed for two diseases: glaucoma and diabetic retinopathy. The model takes into account digital data of ophthalmological equipment obtained from the optic nerve disk and macular area. Criteria that take into account the thickness of the macular zone and the parameters of the optic nerve disc were developed (integral volume and area of the neuroretinal rim, the area of the optic disc excavation). The proposed mathematical model gives the opportunity to predict the development of combined pathology in relation of the integral area of the neuroretinal rim to the integral volume, as well as to a criterion that takes into account the thickness of the macula and the area of excavation of the optic nerve head. The model makes it possible to give a clear risk of developing diseases (low, high) and a significant prognosis of the course of diseases (good prognosis for vision, bad prognosis for vision). The capability of the mathematical model to predict the development of the disease in a patient with type 2 diabetes is demonstrated.
\end{abstract}

\section{Introduction}

The problem of the relationship between primary open-angle glaucoma and diabetes mellitus has been studied worldwide for more than eighty years [1-4]. Currently, the common pathogenetic mechanisms of the two diseases have been proven. Both glaucoma and diabetic retinopathy affect the internal parts of the retina [5-7].

Oxidative stress, hyperglycemia, hypoxia with the release of angiogenic factors play a crucial role [9-12]. As a result of increased intraocular pressure, the optic disc suffers with mechanical compression of its fibers in the ethmoid plate [13-15]. A detailed analysis of the optic nerve disk at the morphological level in the early stages of the disease is provided by a

* Corresponding author: irina.docent2000@mail.ru 
modern optical coherence tomograph [16-20]. The Protocol of analysis of the optic nerve head evaluates the results of measurement of the parameters of the neuroretinal rim. Optical coherence tomography provides objective digital data on the integral area and volume of the neuroretinal rim, the excavation of the optic nerve disk according to the level of retinal pigment epithelium, and also determines the layer of retinal nerve fibers located around the optic nerve disk using circular scans. Both glaucoma and diabetic retinopathy damage the central region of the retina. With glaucoma, Bjerrum scotomas appear in the center, and with diabetic retinopathy, retinal edema occurs in the macular zone. Optical coherence tomography makes it possible to estimate the thickness of the retina in 9 sectors of the macula [21-23].

The search for morphological relationships between the optic nerve disk and the macula in the analysis of optical coherence tomography data is of great scientific interest. The found relationships will help to create mathematical models and computer programs not only for diagnostics, but also for the prognosis of two diseases [24,25]. Mathematical modeling and programming are relevant today not only in medicine, but also in other fields of science [2630].

\section{Problem}

To develop a mathematical model for the prognosis of diabetic retinopathy and primary openangle glaucoma based on studies of the macular zone and the state of the optic nerve disk according to optical coherence tomography.

\section{Materials and methods}

\subsection{Mathematical processing of digital data of the macular zone thickness based on optical coherence tomography data}

To assess the state of the retina, it was decided to develop a criterion that takes into account the thickness of the macular zone. Map of the macula E M5 (figure 1) and a table of thickness of the retina of the macula (figure 2) are the results of the operation of an optical coherent tomography.

A criterion of the influence of retinal thickness, which is characterized by the severity of the volume of edema in the thickness of the retina in 9 sectors of the macular zone, is proposed:

$$
R_{1}=\frac{\sum_{1}^{9}\left(\mathrm{~h}_{i}-\mathrm{h}_{\min }\right)}{\left(\mathrm{h}_{\max }-\mathrm{h}_{\min }\right) \cdot \mathrm{m}} .
$$

where $R_{1}$ - retinal thickness criterion;

$\mathrm{h}_{i}$ - thickness of retinal sectors (in each sector of the macular zone), $\mu \mathrm{m}$;

$\mathrm{h}_{\text {min }}$ - retinal thickness in one of the scanning sectors of the macular zone, excluding the central and extreme sectors, $\mu m$; 
$\mathrm{h}_{\max }$ - maximum retinal thickness of the macular zone, $\mu \mathrm{m}$;

$\mathrm{m}$ - number of retinal scan sectors $(\mathrm{m}=9)$.

\subsection{Mathematical processing of digital data on the state of the optic nerve disk based on optical coherence tomography data}

To assess the state of the optic disc, the following criteria are proposed: integral area of the neuroretinal rim, integral volume of the neuroretinal rim and optic disc excavation area. Sensitivity map (figure 3) and histogram of threshold frequencies (figure 4) are the results of the fundus microperimeter.

It was proposed to determine the inverse value of the average value of the width of the neuroretinal rim as a criterion for evaluating the state of the neuroretinal rim:

$$
\frac{S_{b}}{V_{b}}
$$

where $\frac{S_{b}}{V_{b}}$ - inverse value of the mean value of the width of the neuroretinal rim, $\mathrm{m}^{-1}$;

$S_{b}$ - integral area of the neuroretinal rim, $\mathrm{m}^{2}$;

$V_{b}$ - integral volume of the neuroretinal rim, $m^{3}$.

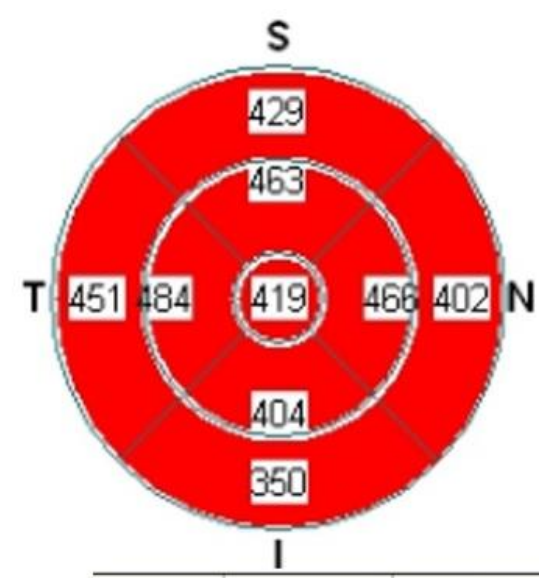

Fig. 1. Map of the macula E MM5 with an information about the thickness of the retina of the macula in fovea, parafovea, perifovea according to the optical coherent tomograph, $\mu m$.

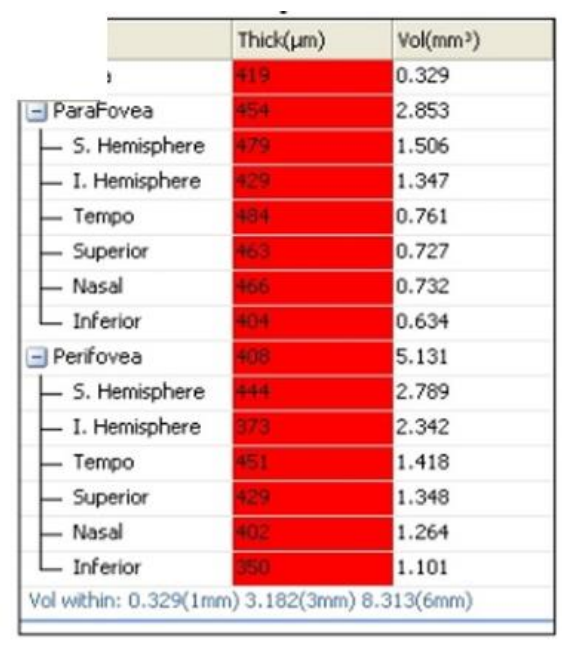

Fig. 2. Table of retinal thicknesses of the macula in fovea, parafovea, perifovea according to the optical coherent tomograph, $\mu m$. 


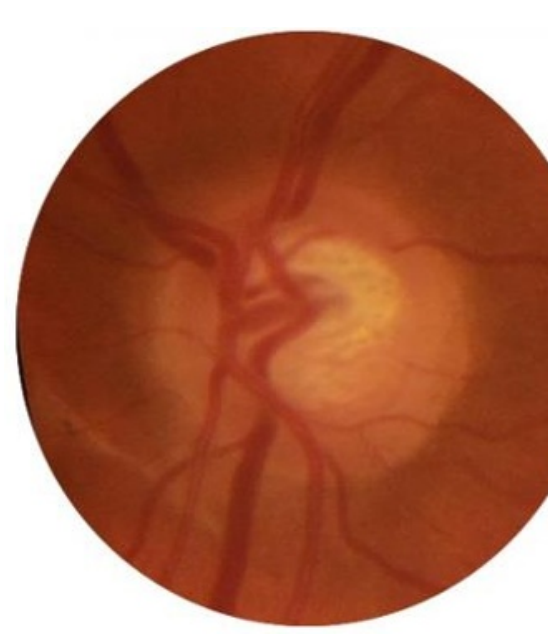

Fig. 3. Photo registration of the optic disc.

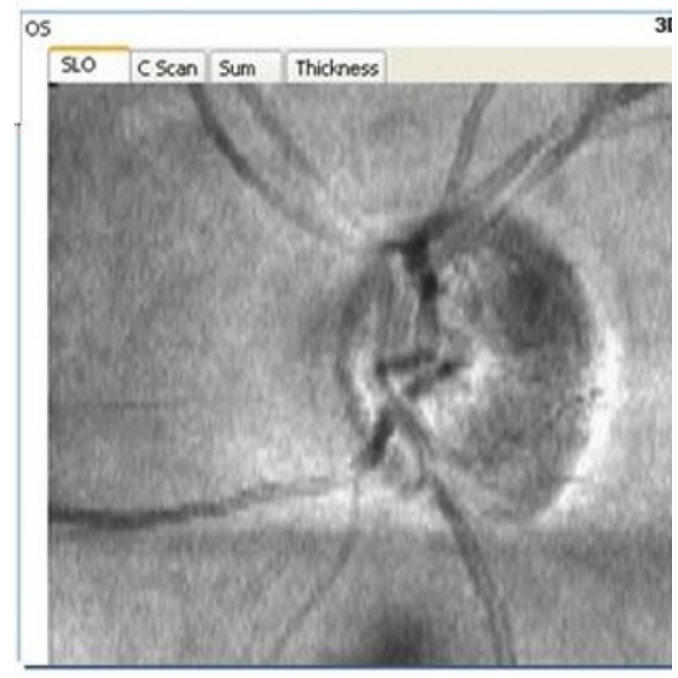

Fig. 4. View of the optic nerve disk by optical coherence tomography.

It is proposed to use the criterion for evaluating the state of the optic nerve disk as a criterion $R_{G D R}$ :

$$
R_{G D R}=\frac{S_{b} \cdot \sqrt{S_{e}}}{V_{b} \cdot R_{1}}
$$

where $R_{G D R}$ - criterion of the primary open-angle Glaucoma and Diabetic Retinopathy;

$S_{e}$ - optic disc excavation area, $m^{2}$.

\subsection{Relationship of the prognosis of diabetic retinopathy and primary open- angle glaucoma with the calculated values of the optic disc}

All developed formulas are based on the method of normalization of the studied indicator. Within the framework of this mathematical mode: $R_{1}$ - the influence of retinal thickness;

$$
\frac{S_{b}}{V_{b}} \text {-influence of the neuroretinal rim; } R_{G D R} \text { - influence of the optic disc condition. }
$$

Based on the results of the research, a table that determines the relationship between the developed criteria and the prognosis of the disease was obtained (table 1). 
Table 1. The prognosis of the disease depending on the criterion $R_{G D R}$ and $\frac{S_{b}}{V_{b}}$.

\begin{tabular}{|c|c|c|c|c|}
\hline$R_{G D R}$ & $\frac{S_{b}}{V_{b}}$ & $\begin{array}{c}\text { Disease } \\
\text { diagnosis }\end{array}$ & $\begin{array}{c}\text { Risk of } \\
\text { progression }\end{array}$ & $\begin{array}{c}\text { Disease } \\
\text { prognosis }\end{array}$ \\
\hline $3 \leq R_{G D R}<5.5$ & $\frac{S_{b}}{V_{b}}<3.5$ & $\begin{array}{c}\text { Stage 1 } \\
\text { glaucoma }\end{array}$ & Low & $\begin{array}{c}\text { Good prognosis } \\
\text { for vision }\end{array}$ \\
\hline $5.5 \leq R_{G D R}<10$ & $3.5 \leq \frac{S_{b}}{V_{b}}<5$ & $\begin{array}{c}\text { Stage 2 } \\
\text { glaucoma }\end{array}$ & High & $\begin{array}{c}\text { Good prognosis } \\
\text { for vision }\end{array}$ \\
\hline$R_{G D R} \geq 10$ & $\frac{S_{b}}{V_{b}} \geq 5$ & $\begin{array}{c}\text { Stage 3 } \\
\text { glaucoma }\end{array}$ & High & $\begin{array}{c}\text { Bad prognosis } \\
\text { for vision }\end{array}$ \\
\hline
\end{tabular}

\section{Results and discussion}

Based on the proposed mathematical model, a computer program for calculating the prediction of the progression of diabetic retinopathy and primary open-angle glaucoma was created. The initial data for working with the computer program are: macular light sensitivity in 9 sectors of the macular zone scan, as well as parameters of the optic nerve disk (integral area of the neuroretinal rim, integral area of the neuroretinal rim, optic disc excavation area). The result of the program is the prognosis of the progression of diabetic retinopathy and primary open-angle glaucoma.

To debug the program, a patient with diabetic retinopathy and primary open-angle glaucoma was examined.

The patient examination data are presented by an optical coherent tomography (table2).

Table 2. Retinal thickness of the examined patient in the macula (fovea, parafovea, perifovea) according to the optical coherence tomograph data

\begin{tabular}{|c|c|c|}
\hline \multicolumn{2}{|c|}{ Section } & Thick, $\boldsymbol{\mu m}$ \\
\hline \multicolumn{2}{|c|}{ Fovea centralis } & 419 \\
\hline \multirow{4}{*}{ ParaFovea } & Tempo & 484 \\
\cline { 2 - 3 } & Superior & 464 \\
\cline { 2 - 3 } & Nasal & 466 \\
\cline { 2 - 3 } & Inferior & 404 \\
\hline \multirow{4}{*}{ PeriFovea } & Tempo & 451 \\
\cline { 2 - 3 } & Superior & 429 \\
\cline { 2 - 3 } & Nasal & 402 \\
\cline { 2 - 3 } & Inferior & 350 \\
\hline
\end{tabular}

Data on the state of the patient's optic nerve disk are presented (table.3) 
Table 3. Data on the state of the patient's optic nerve disk.

\begin{tabular}{|c|c|}
\hline Optic disc parameters & Value \\
\hline Integral area of the neuroretinal rim, $m^{2}$ & 1.732 \\
\hline Integral volume of the neuroretinal rim, $m^{3}$ & 0.253 \\
\hline Optic disc excavation area, $m^{2}$ & 1.242 \\
\hline
\end{tabular}

As a result of the calculation, the program determined the value of the criterion $R_{G D R}=12.72$ as well as the inverse value of the thickness of the neuroretinal rim $\frac{S_{b}}{V_{b}}=6.85$ based on the diagnosis of stage III glaucoma, a high risk of disease progression and a poor prognosis for vision.

The risk of disease progression obtained under the program coincides with the clinical prognosis of the disease made by an ophthalmologist.

Thus, the proposed mathematical model makes it possible to make a prognosis of diabetic retinopathy and primary open-angle glaucoma simultaneously, both from digital ophthalmological data of the macular zone scanning results and from the examination of the optic nerve disk state. This approach provides more opportunities in comparison with separate studies of fundus structures. The use of a computer program helps to create a system for supporting decision-making by an ophthalmologist, as well as optimize the patient's examination time.

\section{Conclusion}

A mathematical model has been created for predicting the development of the combined pathology of diabetic retinopathy and primary open-angle glaucoma. The model makes it possible to provide a clear risk of developing diseases (low, high) and a significant prognosis of the course of diseases (good prognosis for vision, bad prognosis for vision). The model takes into account the clinical and morphofunctional data of the retina, namely, the thickness of the retina in the most important 9 sectors of the macula center, the integral volume of the neuroretinal rim and its area, and the data on the area of the optic disc excavation. The given list of data was combined into a single generalizing criterion $R_{G D R}$ and the ratio of the integral area of the neuroretinal rim to its integral volume $\frac{S_{b}}{V_{b}}$.

The model is designed for processing a large amount of digital data: digital results of optical coherence tomography for the analysis of the optic nerve disk and the central zone of the retina.

The proposed mathematical model for predicting the development of the disease is of great importance for practical health care, as it prevents the development of blindness and low vision in patients with severe form of glaucoma and diabetic retinopathy. This is especially important for patients with glaucoma. Glaucoma has an asymptomatic course and rapid vision loss. The growing intraocular pressure in the eye has a detrimental effect on the fibers of the optic disc, causing the development of compression of axons in the ethmoid 
plate with the appearance of apoptosis. The proposed model takes into account changes in the structure of the optic nerve disk and provides a prognosis for the development of the disease at an early stage, which allows the patient to preserve vision.

The computer program developed according to the model provides a quick high-quality forecast of the development of the disease and approaches each patient in a personalized way.

The prospect for the development of this scientific direction is the creation of an analysis system and an approach algorithm in the diagnosis and prognosis of combined pathologies of the fundus.

\section{References}

1. Amin J, Sharif M, Rehman A, Raza M and Mufti M R 2018 Microscopy Research and Technique 81(9) 990

2. Kaya C, Erkaymaz O, Ayar O and Ozer M 2018 Chaos Solitons and Fractals 113164

3. Sorrentino F S, Matteini S, Bonifazzi C, Sebastiani A and Parmeggiani F 2018 Eye 32(7) 1157

4. Wang W and Lo A C Y 2018 International Journal of Molecular Sciences 196

5. Vorobyeva I V, Moshetova L K and Shcherbakova E V 2017 Oftalmologiya 14(1) 40

6. Vorobyeva I V 2017 Oftalmologiya 14(1) 67

7. Vorobyeva I V 2018 Oftalmologiya 15(2) 189

8. Gaonkar B, Prabhu K, Rao P, Kamat A, Addoor K R and Varma M 2020 Biomarkers 25(5) 397

9. Li X, Li C, Bai D and Leng Y 2021 Neural Regeneration Research 16(1) 172

10. Song S, Yu X, Zhang P and Dai H 2020 Journal of Diabetes and Its Complications 349

11. Mylona I and Tsinopoulos I 2020 Cureus 12(8) e10043

12. Paul S, Ali A and Katare R 2020 Journal of Diabetes and Its Complications 34(8) 107613

13. Abdullah A S, Rahebi J, Ozok Y E and Aljanabi M 2020 Medical and Biological Engineering abd Computing 58(1) 25

14. Karkuzhali S and Manimegalai D 2019 Biocybernetics and Biomedical Engineering 39(3) 753

15. Qureshi I, Ma J and Abbas Q 2019 Symmetry-Basel 11(6) 749

16. Zechel F, Kunze R, Konig N and Schmitt R H 2020 TM-Technisches Messen. 87(6) 404

17. Ammar F, Mahjoub A, Ben Abdesslam N, Knani L, Ghorbel M and Mahjoub H 2020 Annals of Medicine and Surgery 54125

18. Arf S, Muslubas I S, Hocaoglu M, Ersoz M G, Ozdemir H and Karacorlu M 2020 Graefes Archive for Clinical and Experimental Ophthalmology 258(6) 1165

19. Lawman S, Williams B M, Zheng Y and Shen Y 2020 Measurement Science and Technology 31(6) L065230

20. Leal-Gonzalez M, Pessanha F, Gonzalez-Oliva M A, Perez-Fernandez E and Gili P 2020 Clinical and Experimental Ophthalmology 48(6) 775

21. Ishikawa K, Kohno R, Mori K, Murakami Y, Nakao S, Akiyama M, Yoshida S and Sonoda K 2019 Graefes Archive for Clinical and Experimental Ophthalmology 258(3) 621 
22. Minami Y, Song Y, Ishibazawa A, Omae T, Ro-Mase T, Ishiko S and Yoshida A 2019 Scientific Reports 93703

23. Vidyashree H T, Pasha S M and Rathi S 2018 International Journal of Ayurvedic Medicine 9(3) 219

24. Choi J, Kwon J, Shin, J W, Lee J, Lee S and Kook M S 2017 Plos One 12(9) e0184948

25. Rhodes L A, Huisingh C E, McGwin G, Girkin C A and Owsley C 2019 Journal of Glaucoma 28(6) 481

26. Zubarev K P and Gagarin V G 2021 Advances in Intelligent Systems and Computing (International Scientific Conference Energy Management of Municipal Facilities and Sustainable Energy Technologies EMMFT 2019) 1259593

27. Gagarin V G, Akhmetov V K and Zubarev K P 2018 MATEC Web of Conferences (SPbWOSCE-2017) 17003014

28. Zubarev K P and Gagarin V G 2018 IOP Conference Series: Materials Science and Engineering (International Multi-Conference on Industrial Engineering and Modern technologies) 463032082

29. Gagarin V G, Akhmetov V K and Zubarev K P 2020 IOP Conference Series: Materials Science and Engineering (International Science and Technology Conference "FarEastCon-2019") 753022046

30. Gagarin V G, Akhmetov V K and Zubarev K P 2020 IOP Conference Series: Materials Science and Engineering (International Science and Technology Conference "FarEastCon-2019") 753022045 